\title{
Germanica
}

\section{Literarische Erziehung im Zeichen philosophischer Heiterkeitsdebatten. Auf- und Entheiterung nach}

\section{5}

L'éducation littéraire sous le signe des débats philosophiques sur l'allégresse.

Flux et reflux d'allégresse après 1945

Literary Pedagogics in the context of philosophical debates on cheerfulness since 1945

Stefan Born et Tanja Angela Kunz

\section{CpenEdition}

Journals

Édition électronique

URL : http://journals.openedition.org/germanica/5382

DOI : $10.4000 /$ germanica. 5382

ISSN : 2107-0784

Éditeur

Université de Lille

Édition imprimée

Date de publication : 31 décembre 2018

Pagination : 71-90

ISBN : 978-2-913857-42-1

ISSN : 0984-2632

\section{Référence électronique}

Stefan Born und Tanja Angela Kunz, "Literarische Erziehung im Zeichen philosophischer Heiterkeitsdebatten. Auf- und Entheiterung nach 1945", Germanica [Online], 63 | 2018, Online erschienen am: 01 Januar 2021, abgerufen am 22 Januar 2021. URL: http://journals.openedition.org/ germanica/5382 ; DOI: https://doi.org/10.4000/germanica.5382 


\title{
Literarische Erziehung im Zeichen philosophischer Heiterkeitsdebatten. Auf- und Entheiterung nach 1945
}

\author{
Stefan BorN, Tanja Angela KUNZ \\ Humboldt-Universität zu Berlin
}

\begin{abstract}
Zu Beginn des Jahrtausends wurde das Phänomen ubiquitärer, nicht immer tiefsinniger Heiterkeit unter dem Stichwort der Spaßgesellschaft diskutiert. „Ernste Kommunikation“ schien allgemein unwahrscheinlich gewordenzu sein ${ }^{1}$. Auchin derErziehungstheorie wurde Heiterkeit thematisiert: Heinz-Elmar Tenorth etwa hofft, aus der Beobachterperspektive, die „offen-heitere“ Haltung des Kynismus wieder in die pädagogische Reflexion einführen zu können ${ }^{2}$. Sogar aus der in der Tradition Kritischer Theorie stehenden Erziehungswissenschaft heraus finden sich wohlwollende Bekundungen zur Heiterkeit: Andreas Gruschka jedenfalls hat ein ganzes Buch über den ,heiteren Ernst der Erziehung“ in der Malerei geschrieben ${ }^{3}$. Gruschka und Tenorth fokussieren insofern ,Epochenbrüche', als sie die moderne Pädagogik seit dem Beginn des bürgerlichen Zeitalters in einen Gegensatz zur Heiterkeit stellen,
\end{abstract}

1. - Vgl. Dirk Baecker, „Ernste Kommunikation“, Merkur 51 (1997), H. 579, S. 486-495.

2. - Heinz-Elmar Tenorth, „Zynismus - oder das letzte Wort der Pädagogik“, Zeitschrift für Pädagogik 47 (2001), H. 4, S. 449.

3. - Andreas Gruschka, Der heitere Ernst der Erziehung. Jan Steen malt Kinder und Erwachsene als Erzieher und Erzogene, Wetzlar, Büchse der Pandora, 2005.

GERMANICA, 2018, LXIII, pp. 71 à 90. 
die nun gerade, sozusagen nach einem langen Schlummer, neu entdeckt und wiederbelebt werden müsse. Richtig daran ist, dass Heiterkeit in der Geschichtsschreibung der Pädagogik bislang kein großes Thema war. Hier differieren die Interessen der Erziehungswissenschaft von jenen der Literaturgeschichtsschreibung, für die insbesondere Petra Kiedaischs Studie Ist die Kunst noch heiter? von 1997 einschlägig ist ${ }^{4}$. Andererseits finden sich, auch im 20. Jahrhundert, durchaus pädagogische Anwälte der Heiterkeit, so dass sich in Anlehnung an Kiedaisch auch hier von Phasen der Auf- und Entheiterung sprechen lässt. Auch die Ideengeschichte der Erziehung im 20. Jahrhundert müsste dann Gestalten des Heiteren enthalten, die ähnlich analysierbar und beschreibbar sind wie diejenigen in der Literaturgeschichte. Und was Tenorth und Gruschka registrieren, wäre dann nichts anderes als der Beginn einer neuen Phase der Aufheiterung.

Im Folgenden werden wir in der Erziehungstheorie der Nachkriegsjahrzehnte in der BRD nach Gestalt(ung)en des Heiteren suchen; dabei nehmen wir insbesondere die Literaturpädagogik bzw. die Literaturdidaktik in den Blick, weil wir davon ausgehen, dass hier, in der direkten Auseinandersetzung mit literarischen Konfigurationen des Heiteren, differenzierte Standpunkte entwickelt werden. Kiedaisch hat in ihrer Untersuchung die These vertreten, dass sich bis 1967 Heiterkeitskonzeptionen in der Literatur finden lassen, die sich ,zur Melancholie, zum Ernst als Komplement bekennen und der Vergangenheit nicht beschönigend, sondern kritisch gegenüberstehen"5, bevor die poetischen Heiterkeitsauffassungen durch die schrille Debatte zwischen Adorno und Staiger in einen Degradationsprozess hineingezogen worden seien. $\mathrm{Zu}$ überprüfen wäre nun, wie in dieser Phase parallel zum - und oft genug auch bezogen auf den - literarischen Diskurs die Heiterkeit in der Theorie der Erziehung aufgefasst und ,gestaltet" wurde.

Sowohl mit der Konzentration auf die Zeit nach 1945 bis in die ,langen 60er Jahre' als auch mit der Rede von den Konfigurationen oder auch ,Gestalten' des Heiteren nehmen wir Impulse der Studie

4. - Petra Kiedaisch, Ist die Kunst noch heiter? Theorie, Problematik und Gestaltung der Heiterkeit in der deutschsprachigen Literatur nach 1945, Tübingen, Niemeyer, 1996, S. 275. Darin sammelt sie eine Vielzahl von Belegstellen und stellt damit den Facettenreichtum von Heiterkeitskonzeptionen in Kunst, Philosophie und Gesellschaft seit 1945 heraus. Aus systematischer Perspektive ist Kiedaischs Studie auch deshalb zentral, weil sie mit dem bis dahin vorherrschenden essayistischen Umgang mit dem Thema Heiterkeit bricht. Vgl. dazu Peter Eichhorn, Kritik der Heiterkeit, Heidelberg, Lambert Schneider, 1973; Odo Marquard, „Exile der Heiterkeit“, in: Wolfgang Preisendanz/Rainer Warning (Hrsg.), Das Komische, München, Fink, 1976; Harald Weinrich, Kleine Literaturgeschichte der Heiterkeit, München, C.H. Beck, 2001.

5. - P. Kiedaisch, Ist die Kunst noch heiter?, a.a.O., S. 254. 
Kiedaischs auf ${ }^{6}$, führen diese aber weiter. Allgemein gehen wir davon aus, dass Heiterkeit ein Verhältnis zur Wirklichkeit ist, in dem mit Negativem ,auskömmlich' gelebt werden und das eine Perspektive der Zuversicht und der Versöhnung einschließen kann. Es ergibt sich aus einer Wahrnehmung, die aus Einzelelementen ein übersummatives Ganzes entstehen lässt, das man auch ,gestalthaft' nennen kann. Für unsere Zusammenhänge bietet sich diese Auffassungsweise auch deshalb an, weil der Gestaltbegriff die Grenzen zwischen aisthesis und poiesis, zwischen Theorie, Wissenschaft und Kunst seit Goethe transzendiert ${ }^{7}$ und deshalb auch auf ein disziplinär so hybrides Feld wie die Literaturpädagogik oder Erziehungsphilosophie appliziert werden kann.

Wenn es stimmt, dass die Erziehungswissenschaften um 2000 oder bereits um 1989 in eine Phase der Aufheiterung getreten sind, liegt der Gedanke nahe, dass sich in einer Ideengeschichte der Erziehung, ähnlich wie in der Literaturgeschichte, Konjunkturen des Heiteren aufweisen lassen. Auch die zitierten Theoretiker verorten sich selbst mal mehr, mal weniger ausdrücklich an epochalen Schwellen zwischen Moderne und Post- oder Spätmoderne. Sicherlich ist es riskant, aus Einzelstudien einen generellen Trend abzuleiten, was sich auch an der Studie Kiedaischs zeigt; denn zu einer epochenorientierten Abstraktion steht die individuelle Beschäftigung einzelner Autoren mit differierenden Denkkonzepten von Heiterkeit stets in einem Spannungsverhältnis. Die Ausnahmen lassen die Regel verblassen. Gleichwohl scheint es plausibel, von einem Konnex von Krisenerfahrung und Heiterkeit zu sprechen $^{8}$. So werden die historischen Einschnitte um 1945, 1968 oder 1989 zu Anlässen, Heiterkeitskonzepte zu entwerfen oder zu diskutieren.

Eine Geschichte erziehungswissenschaftlicher und didaktischer Heiterkeitskonzeptionen stellt, wie oben bereits angedeutet, noch ein Desiderat dar. Indem wir einen ersten Anstoß zu solch einer Geschichte geben, hoffen wir, eine neue und aufschlussreiche Perspektive auf pädagogische Konzeptionen der jungen Bundesrepublik zu gewinnen und, womöglich, auch etwas über den aktuellen Heiterkeitstrend in Erfahrung zu bringen.

6. - Vgl. auch P. Kiedaisch, Ist die Kunst noch heiter?, a.a.O., S. 23-31. Sie versteht Heiterkeit als spielerisch-entlastendes Verhältnis zu einer Wirklichkeit (1), die Ernstes und Düsteres (2) ebenso wie eine gestalthafte Wahrnehmungsqualität (3) einschließt.

7. - Vgl. Annette Simonis, ,,Gestalt‘ als ästhetische Kategorie“, in: Jonas Maatsch (Hrsg.), Morphologie und Moderne, Berlin/Boston, de Gruyter, 2014, S. 245-266, bes. S. 253.

8. - P. Kiedaisch, Ist die Kunst noch heiter?, a.a.O., S. 258. 


\section{Rekapitulation von Entwicklungslinien und Hintergrüinden}

In den „langen 60er Jahren“ wurden in der BRD allmählich Ordnungsentwürfe der politischen Romantik und antimoderne Deutungsmuster zugunsten eines westlichen oder universalistischen Liberalismus zurückgedrängt ${ }^{9}$. In diesem Zusammenhang trat auch schrittweise das Spannungsverhältnis ins öffentliche Bewusstsein, das sich aus den Erneuerungsbestrebungen und dem konservativen Beharrungsvermögen in den Kultusbehörden und Lehrerverbänden ergab. Dabei wurde deutlich, dass es auch im Erziehungssystem eine sogenannte „Stunde Null“ nie gegeben hatte. Allein der immense Lehrerbedarf nach 1945 machte eine Besetzung der Stellen mit mehr oder minder belasteten Lehrkräften notwendig, so dass bedeutende personelle Kontinuitäten blieben ${ }^{10}$. Nach 1945 wurden große Teile des Erziehungssystems von jener Generation getragen, die durch zwei Weltkriege geprägt war. Spätestens fünf Jahre nach Kriegsende waren viele NS-Sympathisanten oder -Aktive unter dem Deckmantel teils christlicher, teils liberaler oder konservativer Ideen zurück im Dienst ${ }^{11}$. In der Folge lag der Fokus vor allem auf Kontinuität und Überwindung, was sich, auf literaturwissenschaftlichem Gebiet, in der Methodik (Immanenz), in der literarischen Kritik und ebenso in der Kanonisierungspraxis bis weit in die 1960er Jahre zeigt ${ }^{12}$. Kulturgeschichtlich und auch pädagogikgeschichtlich standen die 1950er Jahre im Zeichen einer moderneskeptischen Fortschreibung älterer Traditionsbestände. Zentral wird insbesondere die Idee ,abendländischer" Wurzeln, auf die eine Rückbesinnung erfolgen müsse.

So griff auch die Erziehungswissenschaft auf Konzepte der Weimarer Republik zurück. Die Geisteswissenschaftliche Pädagogik leistete in den Jahren nach Kriegsende einen Beitrag zur Revitalisierung klassischer und romantischer Literatur- und Heiterkeitskonzepte. Heiterkeit und Frohsinn hatten bei Herman Nohl und Wilhelm Flitner, zentraler

9. - Vgl. Anselm Doering-Manteuffel, „Eine neue Stufe der Verwestlichung? Kultur und Öffentlichkeit in den 60er Jahren“", in: Axel Schildt/Detlef Siegfried/Karl Christian Lammers (Hrsg.), Dynamische Zeiten. Die 60er Jahre in den beiden deutschen Gesellschaften, Hamburg, Christians, 2000, S. 661-672, bes. S. 311-316.

10. - Vgl. Wolfgang Haßberg/Manfred Seidenfuß, ,Modernisierung im Umbruch. Ein Rückblick“, in: Dies. (Hrsg.), Modernisierung im Umbruch, Berlin, LIT, 2008, S. 402.

11. - Franz Pöggeler, ,'Erziehung nach Auschwitz’ als Fundamentalprinzip jeder zukünftigen Pädagogik", in: Fritz Hartmut Paffrath (Hrsg.), Kritische Theorie und Pädagogik der Gegenwart. Aspekte und Perspektiven der Auseinandersetzung, Weinheim, Deutscher Studien Verlag, 1987, S. 66.

12. - Vgl. Jost Hermand, Geschichte der Germanistik, Reinbek bei Hamburg, Rowohlt, 1994, S. 114-164. 
noch bei dem Erziehungsphilosophen Otto Friedrich Bollnow, den Charakter von pädagogischen Tugenden, zu denen sich der Einzelne aufschwingen sollte. In der Literaturpädagogik wurde sie v.a. von Robert Ulshöfer vorangetrieben; von Herman Helmers wurde sie bereits kritisch weiterentwickelt und modifiziert. Mit diesen fünf Protagonisten entsteht sicherlich kein vollständiges Bild von der (literatur-)pädagogischen Theorielandschaft 1945-1967; aber sie alle waren einflussreich und insofern repräsentativ. Dies zeigt nicht zuletzt das Beispiel Bernhard Bergmanns, der diese fluktuierenden Ideen aufgriff und sie in konkrete Bildungspolitik umzusetzen suchte.

Gegen Ende der 1960er Jahre kündigt sich ein grundlegender Wechsel der maßgebenden Theorie an. Die Kontroverse um Emil Staigers Ende 1966 gehaltene Rede zur Verleihung des Zürcher Literaturpreises, auf die u.a. Adorno mit seinem berühmten Aufsatz „Ist die Kunst heiter?“ reagiert hatte, bildet einen prominenten Kulminationspunkt der sich in diesen Jahren zuspitzenden Debatten. Vor Adornos Absage an Heiterkeit in Kunst war bereits ein philosophischer, Trend“ in den Fokus seiner Kritik gerückt: das zum Jargon herabgefallene Begriffsarsenal des Existenzialismus mit seinen „Heilsformeln“13. Als einen der Gewährsmänner dieses Jargons nimmt Adorno zuallererst Bollnow und dessen „Seinsgläubigkeit"14 ins Visier und schreibt sich damit in den heiterkeitstheoretischen Diskurs der Pädagogik dieser Jahre ein.

Die Argumente Adornos werden über die 1960er Jahre hinaus aufgegriffen und leiten damit das vorläufige Ende einer Pädagogik ein, die die Erziehung zur Heiterkeit als ihre, mehr oder weniger unhinterfragte, Aufgabe begreifen kann. Der Weg bis dahin soll im Folgenden ausführlicher beschritten werden.

\section{Heitere Erziehungsphilosophie}

Herman Nohl hat sich von den Vertretern der Geisteswissenschaftlichen Pädagogik am ausführlichsten mit einer Theorie der Kunst und einer Theorie der ästhetisch-literarischen Erziehung beschäftigt. Zwar entwickelte er diese zu großen Teilen bereits deutlich vor 1945, doch er und die Schule der Geisteswissenschaftlichen Pädagogik blieben bis etwa 1970 ,die herrschende Richtung“15, so dass auch auf ältere Schriften zurückgegriffen werden kann, um die theoretische Situation nach 1945 zu erläutern.

13. - Theodor W. Adorno, „Jargon der Eigentlichkeit“, in: Ders., Gesammelte Schriften, Bd. 6, hrsg. von Rolf Tiedemann, Frankfurt a.M., Suhrkamp, 1997, S. 467.

14. - Ebd., S. 429.

15. - Herwig Blankertz, Die Geschichte der Pädagogik, Wetzlar, Büchse der Pandora, 1982, S. 258. 
Nohls Überlegungen stehen im Rahmen einer allgemeinen Theorie des nationalen Kulturprozesses, den er ,die deutsche Bewegung“ nennt und die sich in der Zeit von 1770 bis 1830 formiert haben soll ${ }^{16}$. Die Kunst, aber auch die Philosophie, haben in diesem Rahmen die Aufgabe, dem deutschen Geist zu einem einheitlichen und moralischen Ausdruck zu verhelfen und so an der Verwirklichung und „Verklärung“ des deutschen Lebens mitzuwirken. Den Ursprung dieser Bewegung sieht Nohl bei Herder. Dessen ganze Philosophie ist bei Nohl nichts anderes als der Ausdruck einer Heiterkeit, die im Übrigen typisch für die ersten Generationen der deutschen Bewegung im Ganzen gewesen sei: In Herders Werken sei ,eine reine Heiterkeit und ein Lebensmut, der allen Jammer der Erde hinter sich läßt“; alle seine Werke seien „getragen und beseelt von einem himmlischen Optimismus". Damit aber stehe er „,nicht allein in seiner Zeit, der Optimismus ist vielleicht das tiefste Ethos aller ihrer besten Geister [...]. Es war im Grunde dasselbe, was unsere Dichter zu Idealisten machte und besonders Schiller bis zur Ausschließlichkeit nach Griechenland trieb“. Alle traditionsstiftenden Gründungsfiguren der deutschen Bewegung seien durch heitere Überlegenheit und Optimismus gegenüber einer negativen Wirklichkeit zu charakterisieren:

Jene Leute fühlten wahrhaft das Leben nicht so golden und ewig heiter, das Fragmentarische und Kümmerliche des Daseins, vor allem des Menschenherzens mit seinen kleinlichen und häßlichen Zügen war ihnen tiefer bewußt, als vielleicht einem von uns, aber gerade auf diesem Hintergrund von Jammer und Kleinheit leuchtete ihnen jeder lichte Tag der Natur wie die Offenbarung der eigentlichen Güte des Daseins, und jedes edle Gefühl und jede menschliche Hingabe war ein Beweis seiner innersten Harmonie, jedes Gesetz, das den Zusammenhang aller Dinge erwies, eine Sicherung dieses idealistischen Glaubens, und seine stärkste Waffe die Moralität ${ }^{17}$.

Nohl fasst die Kunst als Mittel der ästhetischen Erziehung auf. Aus diesem Grund macht er Herder zwar zur Gründungsfigur, Schiller aber „zur Galionsfigur" der deutschen Bewegung ${ }^{18}$. Wie jener zeichnet sich Schiller durch seinen Idealismus aus, den Nohl den „Idealismus der Freiheit" nennt. Noch stärker als bei Herder betont Nohl bei Schiller, wie sehr er sich nach seiner Kantrezeption mit unverwüstlichem

16. - Daniela Gertz, Die deutsche Bewegung. Der Mythos von der ästhetischen Erfindung der Nation, München, Fink, 2007, S. 39-41.

17. - Alle Zitate Herman Nohl, „Johann Gottfried Herder“ [1905], in: Ders., Die deutsche Bewegung. Vorlesungen und Aufsätze zur Geistesgeschichte von 17701830, hrsg. von Otto Friedrich Bollnow und Frithjof Rodi, Göttingen, Vandenhoeck \& Ruprecht, 1970, S. 76f.

18. - D. Gertz, Die deutsche Bewegung, a.a.O., S. 51. 
Idealismus einer mangelhaften Wirklichkeit entgegengeworfen habe ${ }^{19}$. Die Gewissheit einer höheren Wahrheit und Idealität habe es Schiller erst erlaubt, mit ,einer großartigen Überlegenheit, mit einer fast wilden Freude [...] die Sinnenwelt, das Schicksal des einzelnen, wie der Völker, schwarz in schwarz" 20 zu malen. Am reinsten und ,eigentlichsten“ sei Schiller in der Schilderung des Soldatenlagers im Wallenstein gewesen. Hier, in der Gegenwart des Todes und im „Element des Krieges“, sei es ihm gelungen, ,seine persönlichste Lebenseinstellung zum reinsten, heitersten, fast idyllischen Ausdruck zu bringen“21, „die heitere Überlegenheit und ewige Keckheit der Jugend"22 sprechen zu lassen.

Nohl hat die Schiller-Vorlesung ,zum erstenmal 1920 gehalten, um den aus dem Kriege heimkehrenden Studenten ein Zeugnis zu geben von der Wirklichkeit des deutschen Geistes und ich habe sie dann 1946, als sich die Situation grausamer wiederholte, noch einmal gebracht" 23 . Sowohl 1920 als auch 1946 ging es ihm darum, Heiterkeit in einer Krisensituation als metaphysisches Bewusstsein von Idealität und Freiheit darzustellen, das sich auch gegen die Erfahrung von Krieg, Tod und Zerstörung, Vermassung, Technisierung und Entfremdung durchhält. Die beiden Weltkriege selbst werden dabei als Schicksalsschläge in einem undurchschaubaren historischen Prozess verstanden. Die erzieherische Aufgabe der Kunst sei es, Leitbilder zu generieren, über deren Nachahmung die Kunst „eine gestaltende Macht des Lebens selbst“ werde; insofern diese Gestaltungen von Leitbildern, vom Bewusstsein, von Idealität und Freiheit getragen sind, dürfen sie als ,heitere' gelten.

Auch bei Nohls Kollege Flitner bildet eine geschichtsphilosophisch gestützte Verfallserzählung den Rahmen der pädagogischen Theorie. Die Aufklärung habe zu einer Verkümmerung des kulturellen Erbes, insbesondere zu einer Schwächung der christlichen Religion beigetragen. Am Ende dieses Verfallsprozesses steht nach Flitner ein Verlust der tradierten Lebensformen zugunsten einer uniformierten Masse ${ }^{24}$. In der Nachkriegszeit sieht er diesen Verfallsprozess unverändert voranschreiten. Bei den ,jüngeren pädagogischen Reformbestrebungen“ dagegen handele ,es sich vor allem darum, auch die ritterlichen Tugenden zum Gemeingut zu machen und den einfachen Mann zum Anteil an der Staatsleitung zu berufen" 25 . Die literarische Bildung scheint ihm dazu geeignet, sofern sie sich auf einen Kanon von Werken bezieht, die

19. - Herman Nohl, Friedrich Schiller. Eine Vorlesung, Frankfurt a.M., SchulteBumke, 1954, S. 45.

20. - Ebd., S. 47.

21. - Ebd., S. 75.

22. - Ebd., S. 5.

23. - Ebd.

24. - Ebd., S. 91.

25. - Ebd., S. 87f. 
„auf metaphysisches Vertrauen gegründet“ $\operatorname{sind}^{26}$. Die Literatur soll dem Menschen eine metaphysische Welt sittlicher Werte aufschließen, die durch einen rein verstandesmäßigen Zugang verschlossen bleiben müsste - so könne Sinn ,für die Heiterkeit des Geistes, für den ganzen Kanon der humanistischen Tugenden“" gewonnen werden 27.

In einer permanent gefährdeten und in Verfall begriffenen Welt garantiert das Bewusstsein unverbrüchlicher Werte noch die Möglichkeit von Heiterkeit, durch die hindurch zudem ,deutscher Geist“, „deutsche Bewegung" verwirklicht werden soll. Ihr Spezifikum hat diese Heiterkeit darin, dass sie gerade nicht historisch differenziert ${ }^{28}$; es gibt einzig Ewigkeitswerte und einen undurchsichtigen Verfallsprozess; jene müssen sich gegen diesen behaupten.

Aus der „Hochburg des ritterlichen Menschen“ heraus ${ }^{29}$ wollte Otto Friedrich Bollnow gerade nicht schreiben. Mit seiner Schrift zur Einfachen Sittlichkeit (1947) reagierte er, einst begeisterter Anhänger Hitlers $^{30}$, nach der Niederlage Deutschlands auf die, nun eingeräumte, „Fragwürdigkeit insbesondere einer hochgespannten heroischen Ethik" 31 und versuchte, ein alternatives Ethos und Leitbild bereitzustellen. Dabei ging es ihm um den Rückgriff auf ein ungeschichtliches, aber volkstümliches und darin durchaus auch kulturspezifisches Ethos, das zudem durch ,natürliche Gesundheit“ ausgezeichnet sei ${ }^{32}$.

Die Erziehenden sollen dabei „heiter", junge Heranwachsende „fröhlich“ sein, ${ }^{33}$ da sie sich erst auf dem Weg zur Heiterkeit befänden.

26. - Wilhelm Flitner, „Über den Kanon der literarischen Bildung“ [1954], in: Ders., Gesammelte Schriften, Bd. 10, hrsg. von Karl Erlinghagen, Andreas Flitner und Ulrich Herrmann, Paderborn [u. a.], Schöningh, 1997, S. 168.

27. - W. Flitner, „Naturforschung und Humanismus“ [1949], in: Ders., Gesammelte Schriften, Bd. 10, a.a.O., S. 141.

28. - Vgl. zu dieser allgemeinen Tendenz in der Klassikdidaktik bis in die 1970er Jahre Helmut Hoffacker/Bodo Lecke, „Beliebte Opfer oder Klassiker in der Schule aus Handbüchern für den Deutschunterricht", in: Heinz Ide (Hrsg.), Bestandsaufnahme Deutschunterricht. Ein Fach in der Krise, Stuttgart, Metzler, 1970, S. 29-31.

29. - Gertrud Bienko/Heinz Ide, „Aus der Hochburg des ritterlichen Menschen“, in: H. Ide, Bestandsaufnahme Deutschunterricht, a.a.O., S. 147-169.

30. - Briefdokumente aus den 1930er Jahren belegen die Hingabe Bollnows an den Führer (vgl. Wolfgang Klafki/Johanna-Luise Brockmann, Geisteswissenschaftliche Pädagogik und Nationalsozialismus. Hermann Nohl und seine ,Göttinger Schule 1932-1937, Weinheim/Basel, Beltz, 2002). Bollnow, der seit 1933 Mitglied in Alfred Rosenbergs Kampfbund für deutsche Kultur und seit 1940 Mitglied der NSDAP war, hat während des Dritten Reichs beständig publiziert und erhielt 1946 ohne Entnazifizierungsprozess eine Professur in Mainz.

31. - So im Vorwort zur 2. Auflage von 1956. (O. F. Bollnow, „Einfache Sittlichkeit“", in: Ders., Studienausgabe in 12 Bänden, Bd. 3, Würzburg, Königshausen \& Neumann, 2009, S. 3).

32. - Ebd., S. 15.

33. - Otto Friedrich Bollnow, „Wächst das Rettende?“, Zeitwende 48 (1977), S. 112. 
Diese ist bei Bollnow die Vorstufe zur „Seligkeit“34. Als quasi-religiöse Kategorie steht sie dem Zentralbegriff seiner pädagogischen Anthropologie, der Geborgenheit, nahe. Heimat, Heiterkeit und Geborgenheit lassen sich, wie Bollnow, Heidegger zustimmend, referiert, als versöhnte und erfüllte Stimmungsgründe letztlich wechselseitig substituieren ${ }^{35}$.

Mit zunehmendem Alter werde Geborgenheit/Heiterkeit jedoch problematisch und gleichsam perforiert; sie bleibt dann, wie Bollnow es ausdrückt, ,ein gefährdetes Gut“"36, insbesondere für den modernen Menschen, der wisse, dass sich die Welt nicht restlos behausbar machen lasse, sondern dass dunkle und bedrohliche Aspekte bleiben: „Wir wissen heute“, schreibt Bollnow, dass ,das von uns Überschaute wie der enge Umkreis eines Laternenscheins inmitten eines Ozeans von Dunkelheiten ist" 37 . Doch gerade angesichts der offenkundig bedrohlichen Unheimlichkeit, sei es, nur allzu „billig, fasziniert nur immer wieder die Abgründe anzustarren. Heute erscheint es wichtiger, nach den heilenden und bergenden Kräften auszuschauen, die vielleicht darüber hinwegführen können“"38. Unter Rekurs auf Heideggers philosophische Entwicklung nach Sein und Zeit $^{39}$ verweist Bollnow auf dessen Analogiesetzung von Heilem und Heiterem. Hier, wie in seinem eigenen Verständnis, sieht Bollnow das Heile/Heitere als Zustand einer „durch keinerlei Störung zu vernichtenden inneren Ganzheit und Unversehrtheit" 40 . Die Erkenntnis dieses Heiteren/Heil-Seins und der Zugriff auf ein eigenes Heiteres/Heil-Sein wird bei ihm zur Voraussetzung für ein Erfassen des Heitern/Heil-Seins der Welt als Ganzer ${ }^{41}$. In ihrem Kern sind, nach Bollnow, Welt und Mensch folglich heiter/heil, d.h. geborgen, auch und gerade dann, wenn an der Oberfläche Gefahr, Sinnlosigkeit und Zerstörung herrschen ${ }^{42}$.

34. - O. F. Bollnow, Einfache Sittlichkeit, Göttingen, Vandenhoeck\&Ruprecht, 1947, S. 85. In der Schriften-Ausgabe Bollnows von 2009 ff. fehlt der Abschnitt zur Freude; daher wird hier aus der 1. Ausgabe von 1947 zitiert.

35. - O. F. Bollnow, „Die pädagogische Atmosphäre“ [1964], in: Ders., Studienausgabe in 12 Bänden, Bd. 7, Würzburg, Königshausen \& Neumann, 2013, S. 199.

36. - Ebd., S. 199.

37. - O. F. Bollnow, „Maß und Vermessenheit des Menschen“ [1962], in: Ders., Studienausgabe in 12 Bänden, Bd. 3, a.a.O., S. 159.

38. - O. F. Bollnow, „Der Begriff des Heilen“, Situation. Beiträge zur phänomenologischen Psychologie und Psychopathologie (1954), S. 25.

39. - Die ,lange anhaltende Latenzperiode der Bunderepublik bis zum Beginn der 60erJahre“ ist beherrscht von der Philosophie Heideggers (Jürgen Habermas, „Heidegger - Werk und Weltanschauung", in: Victor Farías, Heidegger und der Nationalsozialismus, Frankfurt a.M., S. Fischer, 1989, S. 13).

40. - O. F. Bollnow, „Der Begriff des Heilen“, a.a.O., S. 19.

41. - Ebd., S. 18.

42. - Ebd., S. 15. 


\section{Klassizistische Heiterkeit}

Nohls und Flitners Modell wurde in der Literaturdidaktik insbesondere von Robert Ulshöfer auf eine für den Deutschunterricht ausgelegte Literaturdidaktik und -methodik appliziert. Die Kontroversen, die er auslöste, spielten, wie er auch selbst rückblickend feststellt, „eine beherrschende Rolle“ in der Geschichte des Deutschunterrichts nach $1945^{43}$. Sein didaktisches Modell hat er bereits in der unmittelbaren Nachkriegszeit entwickelt und in den folgenden Jahrzehnten zwar mehrmals weiterentwickelt, in den Grundzügen jedoch beibehalten. An diesem Modell entzündet sich in den späten 1960er Jahren eine Kritik, die den mentalitätsgeschichtlichen Wandel besonders deutlich macht.

Ulshöfer hatte von Nohl die Vorstellung eines gesetzmäßigen Ablaufes von Phasen in der ,pädagogischen Bewegung“ übernommen, die wiederum in der deutschen Bewegung stehen sollen; von Flitner bezieht er die Vorstellung, es gehe in der Erziehung um die Bereitstellung von ästhetisch-gestalthaften Leitbildern, die dem sittlich zersetzenden Prozess der Aufklärung etwas entgegenstellen können. Solch ein Leitbild erkennt Ulshöfer, auch darin ganz bei Flitner und Nohl, im ,ritterlichen Menschen" ${ }^{44}$. Es versteht sich, dass Literatur, sofern sie keine problematische moderne Dekadenzerscheinung ist, auch „heiter" sein muss, soll sie ein derartiges Leitbild bereitstellen können. Die Literatur des 18. und 19. Jahrhunderts gibt Ulshöfer zufolge Gestalten her, „die bei aller Individualität doch als Leitbilder oder Repräsentanten einer Epoche oder eines Menschentypus gelten können"45. Zum deutlichen Bruch mit diesem Stil komme es mit dem Naturalismus und Expressionismus, die „ein bewußtes Zerstören des Menschenbildes der Vergangenheit“ darstellten ${ }^{46}$.

Zum Verfall des literarischen Leitbildes erstellt Ulshöfer ein Unterrichtsmodell: Der Darstellung der Dorothea in Goethes Hermann und Dorothea soll in didaktischer Absicht u.a. die naturalistische Menschenzeichnung in Hauptmanns Webern entgegengehalten werden. Während es über Dorothea noch heißt: „Frei und heiter zeigt sie des Kopfes zierliches Eirund“ ${ }^{47}$, bemerkt er über die Weber, sie seien „,in

43. - Robert Ulshöfer, Mein Deutschunterricht. Geschichte und Zukunftsperspektiven eines didaktischen Modells, Frankfurt a.M. [u.a.], Peter Lang, 1991, S. 8.

44. - R. Ulshöfer, Methodik des Deutschunterrichts: Mittelstufe II, Stuttgart, Klett, 1957, S. 45.

45. - R. Ulshöfer, „Der Wandel des Menschenbildes in der Dichtung des 19. Jahrhunderts" [1951], in: Ders., Theorie und Praxis des Deutschunterrichts Sekundarstufe II. Aufsätze 1946-1996, Frankfurt a.M. [u.a.], Peter Lang, 1997, S. 177.

46. - Ebd., S. 179.

47. - Ebd., S. 180. 
der Mehrzahl flachbrüstige, hüstelnde ärmliche Menschen mit schmutzigblasser Gesichtsfarbe“"48.

Aus diesem und weiteren Vergleichen zieht Ulshöfer den Schluss, dass ein Degenerationsprozess stattfinde, der nicht etwa durch gesellschaftliche Analyse, sondern durch die philosophische Besinnung auf das klassische Leitbild aufzuhalten sei. Der modernen Literatur dagegen misstraut er, denn sie offeriere eine ungesunde Anthropologie. Mit der Analogiebildung, heiter - gesund" und ihrer Dichotomisierung mit, verkommen - krank ${ }^{6}$ nimmt Ulshöfer zugleich eine Zuschreibungstradition auf, die bis in die Realismusdebatten des 19. Jahrhunderts zurückreicht und auch in Staigers Schelte der Gegenwartsliteratur zur Anwendung kommt ${ }^{49}$.

Die Alternative von klassischer und moderner Literatur aber entspricht für Ulshöfer der Alternative von heiterer und schwarzer Dichtung. Die Gegenwartsliteratur akzeptiert Ulshöfer zwar als Unterrichtsgegenstand, sieht darin jedoch stets eine Konzession an den literarischen Zeitgeist. Gerade die Ausdrucksformen, die aufs NichtHeitere oder "Schwarze“ weisen, das Absurde, Groteske und sogar die unversöhnte Satire und die Ironie werden als Phänomene problematischer Modernität zur Kenntnis genommen. So müsse der Erzieher sich ernsthaft fragen, ob die Jugend ,mit den Augen Thomas Manns oder Franz Kafkas“" sehen lernen solle, anstatt ,mit den Augen Goethes oder Gottfried Kellers" 50 . Alle Formen von Negativität, diejenigen von Kafka und Thomas Mann eingeschlossen, werden von Ulshöfer als Abweichungen von einem Stilideal klassischer „Ganzheit" verstanden, das als ,ganz' auch im Sinne des ,Heilen` begriffen wurde.

In diesem Sinne gab Ulshöfer auch zwei Hefte von „Der Deutschunterricht" zum Thema ,heitere Dichtung in der Schule“ heraus (1962/1966). In den Vorworten erklärt er es zum Ziel, ,dem beseelten Frohsinn, dem geistreich-scherzhaften Spiel der Phantasie, einer, letztendlich ,gesunden', Lebensfreude mehr Raum in der Schule zu schaffen" 51 . Hinter allen Formen des Komischen und Scherzhaften erkennt er eine habituelle Einheit:

[...] das Heitere, das überlegene Spiel der Phantasie mit den Vorstellungen der Wirklichkeit, Spiel des Geistes mit dem Stoff, Spiel

48. - Ebd., S. 189.

49. - Vgl. P. Kiedaisch, Ist die Kunst noch heiter?, a.a.O., S. 154.

50. - R. Ulshöfer, „Die Wirklichkeitsauffassung in der modernen Prosadichtung. Dargestellt an Manns Tod in Venedig, Kafkas Verwandlung und Borcherts Kurzgeschichten. Verglichen mit Goethes Hermann und Dorothea" [1955], in: Ders., Theorie und Praxis des Deutschunterrichts, a.a.O., S. 199.

51. - R. Ulshöfer, „Zur Einführung“, Der Deutschunterricht 14 (1962), H. 5, S. 4. 
der Sprache mit dem Sinn - als Herausforderung an den Menschen, damit er sich und sein Werk nicht zu ernst nehme ${ }^{52}$.

\section{Konfessionelle Heiterkeit}

Bollnow, für den eine große Nähe zwischen religiösen Erweckungserlebnissen und Erweckung im Erziehungsprozess besteht $^{53}$, hat in konfessionell geprägten Kreisen der Nachkriegszeit eine breite, wenn auch stark simplifizierende Rezeption erfahren und damit auch in die konkrete Schulpolitik gewirkt. So plädierte z.B. Bernhard Bergmann, der als Vertreter eines konfessionell ausgerichteten Schulwesens in den 1950er und 1960er Jahren zusammen mit Christine Teusch, Werner Schütz und Paul Mikat für die gesetzlichen Vorgaben der Volksschule in Nordrhein-Westfalen zuständig war, in seinen Leitlinien für eine neue Erziehung von 1949 für die „Rückbesinnung auf die einfachsten sittlichen Grundlagen des Volksdaseins“, sowie für die „Erziehung zu einem vollen Menschentum, zu Ehrfurcht und Treue, zu innerer Wahrhaftigkeit, zum rechten Gebrauch der Freiheit, [...] Schlichtheit und Echtheit, zur sittlichen Arbeitsgesinnung und Pflichtentreue“ 54 .

Der Konsolidierungswille des Bürgertums in der Nachkriegszeit spiegelte sich schulpolitisch in der Wiedereinsetzung des Schichtenmodells, das sich an einem naturgegebenen Begabungsbegriff orientierte. Diese Begabungstypologie war eine aus politischen Interessen der Weimarer Republik hervorgegangene Erfindung der Geisteswissenschaftlichen Pädagogik der 1920er Jahre ${ }^{55}$. Die Wiederaufnahme dieses empirisch nicht begründbaren Postulats zielte nach 1949 auf die Festigung vorhandener sozialer Strukturen und nicht auf die Herausbildung von selbstständigen, kritisch denkenden Schülern. Jene blieben im Wesentlichen dem erzieherischen Leitbild des Bildungsempfängers unterstellt ${ }^{56}$, gegliedert nach dem statischen Modell der Lebenskreis-Bildung, das keinen Aufstieg zwischen den Gruppen zuließ: Demzufolge bildete die Volksschule den volkstümlichen Menschen aus, dem man ein gegenstandsgebundenes, d.h. nicht-abstraktes Denken und ,gemütvolles Erfassen“ beimaß. Die höheren Schulen bereiteten auf einen

52. - R. Ulshöfer, ,Zur Einführung“, Der Deutschunterricht 18 (1966), H. 3, S. 4.

53. - Vgl. O. F. Bollnow, Existenzphilosophie und Pädagogik. Versuch über unstetige Formen der Erziehung, Stuttgart, Kohlhammer, 1959.

54. - Bernhard Bergmann, Leitlinien für eine neue Erziehung, zit. nach: Christopher Schwarz, „Abendland und Arbeitswelt“, in: W. Haßberg/M. Seidenfuß, „Modernisierung im Umbruch. Ein Rückblick“, a.a.O., S. 159.

55. - Vgl. Karl-Heinz Dammer/Elmar Wortmann, Mündigkeit: Didaktische, bildungstheoretische und politische Überlegungen zu einem schwierigen Begriff, Baltmannsweiler, Schneider Hohengehren, 2014, S. 109f.

56. - C. Schwarz, ,Abendland und Arbeitswelt“, a.a.O., S. 166. 
Lebenskreis vor, der eine „abstrakte Geistigkeit“" zuließ ${ }^{57}$. Bergmann ist einer von vielen politisch einflussreichen Repräsentanten dieses vertikalen Modells, das erst im Zuge der Bildungsoffensive der 1960er Jahre durchlässiger wurde.

Für Bergmann geht es bei den Bildungszielen ,nicht zuerst um didaktische und methodische Fragen, sondern um einen neuen Geist“, dessen Grundlage ein ewig gültiger Tugendkatalog zu sein habe ${ }^{58}$. Auch bei ihm zeigt sich im Wesentlichen eine konzeptionelle und begriffliche Kontinuität, die er aus der antidemokratischen Ideenbewegung des Jungkonservatismus der Weimarer Republik bezog 59 und unter veränderten Begrifflichkeiten fortsetzte. So greift Bergmann sein völkisch-katholisches Konzept nach 1945 als katholisch-antikommunistisches wieder auf und orientiert es statt an der Volkwerdung an der Individualaufgabe der Menschwerdung, wobei er die sittlich-religiöse Erneuerung an letztgültige und nicht vom Menschen gesetzte Normen bindet $^{60}$.

Die Bedeutung insbesondere der Ehrfurcht als antirationales Erziehungsziel und ihrer Verbindung zur Scham hatte Bollnow bereits 1942 ausgeführt ${ }^{61}$. Wissenserwerb und Erkenntniszuwachs treten mit dieser pädagogischen Ausrichtung in den Hintergrund. 1958 publiziert Bergmann einen Bildband von fröhlicher Schule mit dem Titel Schulklima vorwiegend heiter. Darin geht er davon aus, dass „frohe und heitere Schule auch heute nicht Theorie, Illusion oder Utopie sind, sondern in aller Schul- und Erziehungsnot reiche, vielgestaltige Wirklichkeit" seien ${ }^{62}$. Auch hier wird das zu erzielende Schulklima mit Begriffen umschrieben, die in unmittelbarer Nähe zur Heiterkeit stehen: Fröhlichkeit, Wärme, Vertrauen. Heiterkeit wird damit zum wesentlichen Merkmal sittlicher Erziehung und zum qualifizierenden Signum „,richtiger" Lehrergesinnung. Zudem wird gelingende Unterrichtspraxis

57. - Bettina Alavi, „Volkstümliche Bildung in der Nachkriegszeit“, in: W. Haßberg/M. Seidenfuß, ,,Modernisierung im Umbruch. Ein Rückblick“, a.a.O., S. 320f.

58. - Bernhard Bergmann, „Volksschulrichtlinien und ihr Sinn“, in: Ders. (Hrsg.), Volksschule heute. Beiträge zur Entfaltung der Richtlinien für die Volksschule des Landes Nordrhein-Westfalen, Ratingen, 1956, S. 21. Bergmann hält am Schichtenmodell und der anthropologisch begründeten Begabungstypologie fest.

59. - Vgl. Klaus Himmelstein, Kreuz statt Führerbild. Zur Volksschulentwicklung in Nordrhein-Westfalen 1945-1960, Frankfurt a.M. [u.a.], Lang, 1986, S. 113.

60. - Ebd., S. 121ff.

61. - Vgl. O. F. Bollnow, „Über die Ehrfurcht“, Blätter für Deutsche Philosophie 16. Jg. (1947), S. 345-369.

62. - Das tatsächliche Vorherrschen eines vornehmlich „heitere[n] Grundklima[s]““ in den zeitgenössischen Schulen (B. Bergmann, Schulklima vorwiegend heiter. Ein Bildband von fröhlicher Schule, Bonn, Athenäum, 1958, S. 4) darf angesichts des in der BRD bis 1973 vorhandenen Züchtigungsrechts für Lehrkräfte an Schulen gegenüber den ihnen zur Erziehung anvertrauten Schülern angezweifelt werden. 
am Grad heiterer Atmosphäre messbar. Erziehung zur Ehrfurcht führt in diesem Verständnis nicht nur notwendig zu „Zucht und Ordnung“63, sondern als Folge ebenso zur Heiterkeit, da mit ihr ein bestimmter Begriff von Arbeit nicht um des Geldes oder Erfolgs Willen, sondern auf der Grundlage einer inneren Haltung der Hingabe an die vorhandene Herrschaftsform einher geht. Heitere Schule ist dann die natürliche Ausdrucksform gelingender Erziehung zur vornehmen inneren Haltung.

\section{Allmähliche Auflösung der Heiterkeit}

Hermann Helmers, der Begründer64 der wissenschaftlichen Deutschdidaktik, beschäftigte sich im Zusammenhang mit der Satire und Groteske mit dem Problem der Geborgenheit65. Damit schließt er einerseits an die Terminologie Bollnows an, denkt diesen andererseits im Geist der 1960er Jahre weiter und lässt ihn teilweise hinter sich. Er interpretiert die Entwicklung des kindlichen Humors so, dass im Laufe der Ontogenese sukzessive die „Verfremdungstoleranz“ zunimmt. Verfremdung wird von Helmers einerseits als elementare poetische Operation begriffen. Sie kann als solche aber, andererseits, auch eine Entfremdung von einem kindlichen Bereich sittlicher Integrität und Geborgenheit durch die Emanzipation von gesellschaftlichen Ordnungsvorstellungen bedeuten. In diesem Fall spricht Helmers von Verfremdung im engeren Sinn und versteht darunter die unversöhnte Satire66:

Was die Verfremdung als Kategorie des Komischen jedoch vom Lachen scheidet, ist das Fehlen eines letzten Glaubens an die endgültige Rückkehr zur alten Geborgenheit. Diese Rückkehr darf die Verfremdung gerade nicht wollen: Sie bleibt - das ist ihr Ziel - absichtlich im Raum der Erschütterung des Weltbildes, macht sich den humoristischen Effekt also zunutze, ohne ihn bis zu seiner Wiederaufhebung durchzustehen 67 .

63. - B. Bergmann, Schulklima vorwiegend heiter, a.a.O., S. 5.

64. - Vgl. Hermann Helmers, „Didaktik der deutschen Sprache als wissenschaftliche Disziplin“, Bildung und Erziehung 19 (1966), S. 436-447.

65. - Dagegen rückt der Begriff „Heiterkeit“ bei Helmers als bloßes Resultat von Komikrezeption in den Blick: Zu den Funktionen des Humors gehöre auch ,jener sozial integrierende Effekt [...], den man gemein vage unter den Begriffen Freude oder Heiterkeit subsumiert“. (H. Helmers, Lyrischer Humor. Strukturanalyse und Didaktik der komischen Versliteratur [1971], Stuttgart, Klett, 21978, S. 8) Das mit „Heiterkeit“" im ambitionierten Bollnow'schen Sinn Gemeinte wird dagegen vor allem unter der Chiffre „Geborgenheit" integriert.

66. - H. Helmers, „Einleitung“, in: Ders. (Hrsg.), Verfremdung in der Literatur, Darmstadt, Wissenschaftliche Buchgesellschaft, 1984, S. 3.

67. - H. Helmers, „Der kindliche Humor und die Kategorien des literarisch Komischen“, Wirkendes Wort 15 (1965), S. 108. Vgl. auch Ders., Sprache und Humor 
Während Helmers die Satire als Übergangsphänomen wertschätzt, da sie die Rückkehr in heitere Geborgenheit und die ,heile Welt der Kindheit als Möglichkeit noch einschloss, sei diese im Falle der Groteske - als Weiterentwicklung der Verfremdung - ganz verschwunden. Die Groteske sei vielmehr „ein Abbild der typischen Geisteshaltung jenes Erwachsenen, der zwar um das weiß, was ,jenseits der Berge' ist, der aber in diesem Wissen immer wieder und fast verzweifelt versucht, einen Rückweg zur grundgeordneten, heiteren Welt der Kindheit zu finden"68. Bei Helmers kann sie daher nur insofern im Unterricht thematisiert werden, als sie Anlass zur Kritik gesellschaftlicher Dekadenz gibt.

Er ist einerseits deutlich stärker als Ulshöfer bereit, die Heiterkeit als ein im Hier und Jetzt zu Realisierendes zu problematisieren und insofern auch Trübes und Schwarzes, zu unterrichten'. Allerdings bleibt auch bei ihm eine letztlich durch die Bollnow'sche Kindheitsanthropologie vermittelte und nicht hinterfragbare Grundannahme, dass Geborgenheit und Heiterkeit als die Gestalten kindlichen Bewusstseins wiederzugewinnen seien. Der Weg zu dieser Rückgewinnung führt bei Helmers durch einen Geschichtsprozess, den er in den 1970er Jahren immer weniger geisteswissenschaftlich und zunehmend materialistisch denken wird69. Bereits vor den 1970er Jahren weist Helmers' Position aber voraus auf eine grundlegende Kritik der restaurativen oder konservativen Heiterkeitsdidaktik.

\section{(Un)Heitere Pädagogik und Philosophie: Inwiefern „Ernst machen'?}

Eben jene Frage, die Adorno 1966 in der Negativen Dialektik radikalisiert stellt, „ob nach Auschwitz sich noch leben lasse“70, hatte Bollnow 1954 längst mit dem heiteren Kern des Daseins bejaht. Als ein beißender Angriff auf den ,philosophisch gestimmten Pädagogen“71 Bollnow lesen sich denn auch Adornos 1964 erhobene Vorwürfe gegen den „Jargon der Eigentlichkeit“.

Hier wendet sich Adorno erstmals explizit gegen Bollnow, v.a. gegen dessen 1955 erschienene Neue Geborgenheit. Bollnow kontert

des Kindes [1965], Stuttgart, Klett, ${ }^{21971, ~ S . ~ 146-153 . ~}$

68. - H. Helmers, Der kindliche Humor, a.a.O., S. 110.

69. - Vgl. Peter Stein, „Politischer Literaturunterricht, Projektunterricht, Kritisches Lesen und ästhetische Erziehung - Kritik der vier literaturdidaktischen Hauptkonzeptionen der 70er Jahre“, in: Ders. (Hrsg.), Wieviel Literatur brauchen Schüler? Kritische Bilanz und neue Perspektiven des Literaturunterrichts, Stuttgart, Metzler, 1980, S. 12.

70. - Theodor W. Adorno, „Negative Dialektik“, in: Ders., Gesammelte Schriften, Bd. 6, a.a.O., S. 355.

71. - T. W. Adorno, ,Jargon“, a.a.O., S. 429. 
erst verspätet, 1968, in „Das Zeitalter des Misstrauens“, das zugleich eine Reaktion auf den Zürcher Streit und auf Adornos Replik „Ist die Kunst heiter?" darstellt. Es ist sicherlich schlüssig, wenn Ulrich Wehner als Grund für Bollnows Zögern eine Bewusstheit für den geringen Spielraum an Revisionen mutmaßt, den Adornos Kritik Bollnow lässt ${ }^{72}$. Vielsagend ist dann allerdings, dass sich Bollnow ausgerechnet 1968 äußert, nachdem mit dem Streit um Staigers Rede das konfrontative Potential konservativer Positionen offen zu Tage getreten ist. Auch Staiger hatte sich in seiner Rede schließlich auf die „Heiterkeit des Schönen“ sowie auf eine „sittliche Gesinnung“ der Dichter vergangener Zeiten berufen, mit welcher sie trotz „verdüsterter Einsamkeit“ in der Lage waren, sich ,im Namen der Menschengemeinschaft" und damit gleichfalls beispielgebend ,zur reinsten Menschheit empor[zu] läuter[n]"73. Bollnows Einmischung in die aus diesen Ansichten Staigers hervorgehenden polemischen Debatten ist als Anzeichen zu werten, dass er die existenzphilosophische Unruhe mit dem kritischen Bewusstsein der 68er assoziiert und durch das grassierende Misstrauen eine überkommene, durchaus (klein-)bürgerlich gedachte, Weltordnung in Frage gestellt sah ${ }^{74}$.

Bollnows verkürzte oder verfälschte Existentialismuskritik wendet sich hauptsächlich gegen Heidegger und von ihm aus gegen den Existenzialismus insgesamt ${ }^{75}$. Heidegger wird unterstellt, dass sich der Mensch in der Existenzphilosophie von der Welt und allem Fremden bedroht sehe. Auf diese Weise würden die Welt und die Fülle des Lebens negativ, sie bildeten eine Barriere, die gerade den sittlichen Aufschwung des Menschen verhindere. Statt zur Heidegger'schen „Entschlossenheit“ führe dies zur Verschlossenheit ${ }^{76}$. Im Gegenzug ging es Bollnow um eine lebensphilosophische Erneuerung. So inszeniert er, nach Ulrich Wehner, einen Richtungsstreit zwischen Lebensund Existenzphilosophie und setzt dabei seine Philosophie der Hoffnung von einer Existenzphilosophie ab, die keine ist ${ }^{77}$.

Adorno nimmt auf diesen Aspekt der Fehldeutung Bezug, wenn er in der Jargon-Schrift bemerkt, es mache keinen wesentlichen Unterschied, „ob man geborgen oder erst einmal ungeborgen auftritt“78. Bollnows Fehlinterpretation macht für Adorno letztlich in beide Richtungen

72. - Ulrich Wehner, Pädagogik im Kontext von Existenzphilosophie. Eine systematische Untersuchung im Anschluss an Eberhard Grisebach, Otto Friedrich Bollnow und Theodor Ballauff, Würzburg, Königshausen \& Neumann, 2002, S. 157.

73. - E. Staiger, „Literatur und Öffentlichkeit“, a.a.O., S. 92.

74. - U. Wehner, Pädagogik im Kontext von Existenzphilosophie, a.a.O., S. 157.

75. - Ebd., S. 150.

76. - Ebd., S. 159f.

77. - Ebd., S. 155.

78. - T. W. Adorno, „Jargon“, a.a.O., S. 432. 
keinen Unterschied, denn da beides auf ein Substantielles ziele und so jede radikale Frage ausschließe, offenbarten beide statt transzendentaler Obdachlosigkeit ein metaphysisches Bedürfnis. Somit „feiert man die Bindungen, anstatt in die Spekulation sich zu stürzen"79.

Da für Adorno das Individuum aufgrund seiner Verschränkung mit der Warengesellschaft deformiert ist und wird, kann ein Rekurs auf Innerlichkeit oder neue Geborgenheit, durch welche sich ein Urvertrauen zu sich und den Verhältnissen wiederherstellen lässt, die Krisen unmöglich bewältigen ${ }^{80}$. Angesicht der offenbaren Übel sei der Jargon so „die erpresserische Zuversicht des verängstigten Bewußtseins"

Für Bollnow hingegen ist die ,[g]roße Angst“, die „das Dasein des gegenwärtigen Menschen“ bestimme, „ein gefährliches Bewußtsein“, das „,die schöpferischen Kräfte des Menschen zu ersticken drohe“82. Ebendiese große Angst sei eine fiktive, nämlich durch den Existenzialismus erzeugte, der dem natürlichen Prozess der ,schirmenden und wiederherstellenden Kräfte der Heilung"83 ein bloßes Teilbild der Welt als katastrophische entgegengestellt habe. Der heitere Mensch ist folglich einer, der sich im Angesicht des beschädigten Lebens an seinen heilen Wesenskern zu erinnern weiß. Da Bollnow die existenzphilosophische Angst zum Einzelphänomen, zu einer Stimmung unter einer Vielzahl von gleichberechtigten Stimmungen macht, kann er sie zu den gedrückten Stimmungen zählen und als defizienten Modus disqualifizieren. Doch bei Heidegger meint Angst weder eine gedrückte Stimmung noch einen Rückzug auf weltlose Innerlichkeit, sondern im Gegenteil eine vorreflexive ursprüngliche Weltoffenheit des welthaften menschlichen Geistes. Erst durch die Befindlichkeit der Angst wird der Mensch bei Heidegger seiner selbst als eines in Verantwortung stehenden Seins gewahr ${ }^{84}$.

Der hypothetischen Setzung einer grundlegenden Harmonie steht jene einer permanenten Gefahr der Disharmonie entgegen. Die Vorstellung von einer unmittelbaren Realisierung des Menschen als Mensch betrachtet Adorno daher als „Ideologie der Entmenschlichung“ innerhalb des Phantasmas eines „Ungeteilten, schützend Geschlossenen“85. Einer Erziehung zu Humanität steht somit eine Erziehung zur Skepsis diametral entgegen ${ }^{86}$. Diese richtet sich bei Adorno vor allem auch

79. - Ebd., S. 434.

80. - Vgl. Fritz Hartmut Paffrath: „Einleitung“, in: Ders. (Hrsg.), Kritische Theorie und Pädagogik der Gegenwart..., a.a.O., S. 12.

81. - T. W. Adorno, „Jargon“, a.a.O., S. 436.

82. - O. F. Bollnow, „Der Begriff des Heilen“, a.a.O., S. 16.

83. - Ebd., S. 17.

84. - U. Wehner, Pädagogik im Kontext von Existenzphilosophie, a.a.O., S. 154f.

85. - T. W. Adorno, „Jargon“, a.a.O., S. 452.

86. - „Erziehung wäre sinnvoll überhaupt nur als eine zu kritischer 
gegen den Zentralbegriff des Heilen bei Bollnow. Der Jargon setze so ein ,[v]orgeblich heiles Leben, als Gegensatz zum beschädigten“87.

Für Adorno ,ernten sie [die Vertreter des Jargons] auf verbrannter Erde“88. Stattdessen will er aus gesellschaftskritischer Perspektive Ernst machen. Bereits 1959 hatte Adorno in „Theorie der Halbbildung“ eine negative Bildungstheorie entworfen und sich damit kritisch gegen eine idealistische Beschwörung von Bildung abgesetzt. Stattdessen plädierte er für eine „Erziehung des Madigmachens“89. In einer solchen seien Ganzheitsideale, wie sie nicht selten Hintergrund positiver Bildungstheorien sind, lediglich „Zinnober“90.

Daher fordert er schließlich: „Erziehung müsste Ernst machen mit einem Gedanken, der der Philosophie keineswegs fremd ist: daß man die Angst nicht verdrängen soll ${ }^{\circ 91}$. Nicht heitere Erziehung also kann das Ziel sein, so ließe sich schlussfolgern, sondern Erziehung im Angesicht der Angst. Von dieser Warte aus wird die ab Mitte der 1980er Jahre aufkommende Kritik zahlreicher Pädagogen nachvollziehbar, denen zufolge eine an Adornos Vorstellungen orientierte Pädagogik ,eindeutig apokalyptische Züge“ erhalte ${ }^{92}$. Bis dahin allerdings wird über die langen 1960er Jahre hinaus die Vorherrschaft der Geisteswissenschaftlichen Pädagogik durch eine sich an der Kritischen Theorie orientierende Kritische Pädagogik abgelöst. Mit Schriften, u.a. von Herwig Blankertz, Paul Heimann, Wolfgang Klafki, Klaus Mollenhauer und Paul Schirbel, erhielten nun die Kritiker der dominierenden Nachkriegskonzeptionen primäre Deutungsmacht, so dass die Pädagogik bereits im Ansatz politisch wurde.

\section{Ergebnisse und Ausblick}

Wir konnten zeigen, dass Heiterkeit in der Pädagogik kein neues Denkkonzept oder Leitbild darstellt, das seit dem bürgerlichen Zeitalter vernachlässigt oder sogar bekämpft wurde. In einigen der einschlägigsten pädagogischen Konzeptionen der bundesdeutschen Nachkriegszeit soll mit Heiterkeit eine Wahrnehmungsweise habitualisiert werden, die sich auf die Gewissheit ewiger und unverbrüchlicher Werte stützen kann. Heiterkeit erscheint fast immer als Attribut einer Lebensform, die die

\footnotetext{
Selbstreflexion.“ (T. W. Adorno, „Erziehung nach Auschwitz“, in: Ders., Erziehung zur Mündigkeit, Frankfurt a.M., Suhrkamp, 1971, S. 89).

87. - T. W. Adorno, ,Jargon“, a.a.O., S. 452.

88. - Ebd., S. 431.

89. - T. W. Adorno, Erziehung zur Mündigkeit, a.a.O., S. 146.

90. - Ebd., S. 118f.

91. - T. W. Adorno, „Erziehung nach Auschwitz“, a.a.O., S. 97.

92. - Franz Pöggeler, ,'Erziehung nach Auschwitz“ als Fundamentalprinzip jeder zukünftigen Pädagogik“, a.a.O., S. 58.
} 
klassisch-romantische Tradition als Teil einer spezifisch abendländischen Überlieferung aufnimmt. Der, letztendlich unhintergehbare, metaphysische Wesenskern des Abendlandes und seiner Tradition soll angesichts einer meist eher makrohistorisch und undifferenziert gedachten Verfalls- und Katastrophengeschichte Distanz und Integrität gewährleisten. Heiterkeit ist somit eng verbunden mit Reflexionen auf eine ideale Weltordnung, in der Heiterkeit zum Refugium vor der drohenden Verzweiflung wird. Der heitere Mensch rettet sich vor den Gräueln der Welt in den Modus der Zuversicht und Gelassenheit, indem er eine stete Perspektive der Versöhnung wahrt. Das Aufkommen von Heiterkeitsdiskursen ist dann zwar durchaus Kennzeichen von Krisen, unter dem Deckmantel harmonisierender Überlegenheit kann es jedoch auch, das zeigen vor allem die Nachkriegsentwicklungen, zur Verdrängung notwendiger Auseinandersetzungen kommen.

Im Zuge der 1960er Jahre wird der Aufheiterungstendenz der Nachkriegszeit gerade aus diesem Grund sukzessive Einhalt geboten. Die Pädagogik nimmt wesentliche Grundzüge der Kritischen Theorie auf und macht damit engagierte Gesellschaftsanalyse und -kritik zu Themen der Erziehung.

In literaturpädagogischen Zusammenhängen wird ,Heiterkeit" in der Nachkriegszeit gerne in die Nähe komischer Ausdrucksweisen gerückt. Während Helmers den Literaturunterricht über den Weg des literarischen Humors für zeitgenössische Gestaltungen des Heiteren öffnet, ergibt sich aus Ulshöfers strenger Orientierung des Deutschunterrichts an der Personbildung durch (Selbst-)Beherrschung und seiner Etablierung einer vom klassischen Ideal geleiteten Geschmackshierarchie ein relativ enger Kreis an praktikablen Gattungen des Heiteren. Ohnehin werden aus dem komikdidaktischen Zugriff auf Heiterkeit die Groteske und das Absurde in der Regel ausgeschlossen.

In der Literatur hingegen kamen Ohnmachtserfahrungen, das Absurde und Groteske, die Melancholie, der Nihilismus und die umfassende Entfremdung zur Darstellung. Von dieser Entwicklung weicht die Pädagogik folglich ab. Trotz der konzeptionellen Öffnung in den 1960er Jahren lässt sich eine „Schwarze Pädagogik“ - übrigens bis heute - nicht ausmachen; vielmehr ist der Begriff seit einer gleichnamigen Publikation Katharina Rutschkys ${ }^{93}$ negativ konnotiert.

Bemerkenswerterweise legen die hiesigen Ergebnisse eine gewisse Gleichgültigkeit der Erziehungswissenschaften gegenüber der zeitgenössischen Literaturproduktion offen, die sie mit den dominierenden Entwicklungen innerhalb der Literaturwissenschaften nach 1945 eint. Während sich die philosophische Pädagogik à la Bollnow allerdings

93. - Katharina Rutschky (Hrsg.), Schwarze Pädagogik, Frankfurt a.M., Ullstein, 1977. 
vorderhand in den kleinen Raum des Alltäglichen zurückzog, versuchte sich die Literaturwissenschaft auf einen ,von allen Schlacken des Alltags gereinigten ,Geis[t]“ “ zu beziehen, dem ein „höchst elitäres Literaturverständnis“ und damit ein „,betont kleiner Kanon des Wertvollen“ zugrunde lag94. Mit dem Rückzug u.a. auf die Literatur der Klassik trafen Nachblüte - der 1920er und frühen 1930er Jahre - und Zeitgeist - in Form des amerikanischen close-reading, der werkimmanenten Analyse und der Kunst der Interpretation - in der Literaturwissenschaft und in ähnlicher Form auch bei den AbendlandPädagogen Nohl, Flitner und Ulshöfer zusammen.

Die Phase der Entheiterung, die auf die Intervention Adornos gegen heitere Kunst und Philosophie folgte, ließ vielleicht den Eindruck entstehen, Erziehung habe mit Heiterkeit abgeschlossen. De facto jedoch steht der pädagogische Heiterkeitsdiskurs der Jahrtausendwende in einer langen bürgerlichen Tradition. Wie Desengagement nimmt sich diese Haltung, ob gewollt oder nicht, gegenüber der entheiterten Erziehungsphase der 1960er Jahre aus. In eine metaphysische Theorie der Geborgenheit oder der Geschichte, wie in der Nachkriegspädagogik, führt dieser Schwenk allerdings, bislang jedenfalls, nicht zurück.

94. - Jost Hermand, „Allmähliche Entzauberung. Emil Staiger und die Marburger Junggermanisten der frühen Adenauer-Ära“, ZfGerm N.F., Bd. 16 (2007), S. 52. 